\title{
Are Australian Regional Hospitals Doing Enough for Coeliac Disease Testing in 2012-2013? A Pilot Study
}

\author{
Shu Wen $\mathrm{Xu}^{1,2}$, Rafiqul Islam ${ }^{1,3,4}$, Arup Bhattacharya ${ }^{1,3, *, i}$ \\ ${ }^{1}$ Goulburn Valley Health, Australia \\ ${ }^{2}$ Townsville Hospital, Australia \\ ${ }^{3}$ Department of Rural Health, the University of Melbourne, Shepparton, Australia \\ ${ }^{4}$ School of Health and Social Development, Deakin University, Australia
}

Copyright $\bigcirc 2017$ by authors, all rights reserved. Authors agree that this article remains permanently open access under the terms of the Creative Commons Attribution License 4.0 International License

\begin{abstract}
Coeliac disease diagnosis requires adequate testing, and insufficient testing may increase complications. This pilot study determined the prevalence of appropriate testing for coeliac disease among eligible in-patients admitted in a regional Australian hospital. A retrospective review of all admissions were conducted between August 2012 and January 2013 in the medical, surgical and geriatric divisions for the patients diagnosed with haematinic-deficiency anaemia due to unspecified causes, protein-energy malnutrition due to unspecified causes and irritable Bowel Syndrome. Secondarily an extended review between February 2012 and February 2013 was also conducted to review all serology test requests for coeliac disease to identify the positive serology testing. We observed that, except for Iron Deficiency Anemia (IDA) and Irritable Bowel Syndrome (IBS), a very small proportion of unspecified anaemia and Protein Energy Malnutrition (PEM) patients were investigated (15.6\% and $11 \%$, respectively) for celiac disease. Invasive testing such as histopathology was requested only for about $52 \%$ of serology positive cases. Low diagnostic test requests for coeliac disease warrants the demand for availability of specialist gastroenterologist services and telemedicine support in regional Australia. Training for practicing doctors and continuing medical education around this may help adequate testing and diagnosis.
\end{abstract}

Keywords Coeliac Disease Testing, Regional Hospital, Australia

\section{Introduction}

Coeliac disease is estimated to affect between $0.5 \%$ and $1 \%$ of the general population[1, 2]. In rural Australia the prevalence of the disease may be high. Many cases remain asymptomatic for a long time and resulting in a delayed or non-diagnosis [3]. The correct diagnosis enables patients to avoid the gastrointestinal and nutritional manifestations by restricting gluten in the diet. Since the symptoms are vague and protean, only a small proportion of cases are identified at any certain time [4]. The remaining patients endure ongoing symptomatic burden, malignancy risk, and in some cases, progressive malnourishment and disability [5]. Currently, the Gastroenterology Society of Australia (GESA) guidelines lacking a formal list of coeliac testing indications[6]. While in the World Gastroenterology Organization (WGO) and National Health Service (NHS), United Kingdom guidelines, clinical indications for iron deficiency, unspecified anaemia, unexpected weight loss or protein energy malnutrition, and irritable bowel syndrome are common, although vitamin B12 or folate deficiency anaemia and osteoporosis were absent from the NHS guidelines [5, 6]. Each guideline listed a long and varied selection of relative indications, for which testing could be performed at the clinician's discretion.

Histology of the proximal small bowel remains the gold standard for diagnosis [7]. The recommended serological test is the IgG-specific anti-transglutaminase assay in the IgA deficient (total IgA) subpopulation, and an IgA-specific assay in all others [8]. Equivocal cases require an isotype-equivalent anti-endomysial assay. A negative result, without ongoing clinical suspicion, obviates invasive endoscopy. All other patients require proximal duodenal histology to definitively diagnose or exclude coeliac disease on the basis of the Marsh Grade. However, the concurrent consultations and investigations are costly, invasive, and inconclusive until coeliac disease is considered.

It is recognised that testing and management for coeliac disease is more prevalent in the community and is done by primary care physicians. This exercise however attempted partially to raise the awareness of the medical staff in considering the diagnosis of coeliac disease in an inpatient setting.

While various guidelines have differing thresholds for testing, the current research focuses on the intersection of 
these sets. Patients that also included for coeliac testing are cryptogenic cases of protein energy malnutrition and haematinic-deficiency anemia [5]. Additionally, the diagnosis of irritable bowel syndrome requires exclusion of coeliac disease [8]. While various guidelines have differing thresholds for testing, the current research focuses on the intersection of these sets. This pilot study therefore aimed to determine the prevalence of coeliac testing among subsets of Goulburn Valley Base Hospital in-patients and determined whether the testing was appropriate.

\section{Methods}

\section{Design, Setting, Procedure and Ethics}

Initially the authors' retrospectively reviewed clinical data recorded in the Health Information System in a rural Victorian hospital, Australia.

The data for this initial clinical review were extracted from a complete list of patients admitted under the general surgical, general medical and geriatric departments at the hospital over a six month period between August 2012 and January 2013. Within each subset, both the prevalence and quality of testing was reviewed. All patients documented with a) Iron deficiency, b) unspecified anaemia, c) unexpected weight loss or protein energy malnutrition, d) irritable bowel syndrome e) vitamin B12 or folate deficiency anaemia) osteoporosis were recorded. These conditions were not always listed in the condition that was responsible for the admission to hospital, nor the principal diagnosis, but they appeared in the clinical record as a significant co-morbidity.

Later, in another review of data between February 2012 and February 2013, the authors documented all positive coeliac serology results for these twelve months from the Hospital's Health Information Department. This extended review also included the follow-up and management that each patient received. The study received necessary ethics approval from the HREC, Goulburn Valley Health, Victoria, Australia.

\section{Exclusion}

Patients with diseases and conditions listed in Table 1, at any point in the preceding five years, were excluded. These criteria broadly cover alternative causes of mal-digestion, including gastric, pancreatic and Hepatobiliary diseases, and Malabsorption, arising from bowel resections and inflammatory bowel disease.
Table 1. Exclusion criteria

\begin{tabular}{|c|c|}
\hline \multirow{3}{*}{ Hepatobiliary } & $\begin{array}{l}\text { Alcohol abuse, Non Alcoholic Fatty Liver Disease } \\
\text { (NAFLD), Hepatitis }\end{array}$ \\
\hline & Cirrhosis, Liver Fibrosis \\
\hline & Cholecystectomy \\
\hline Pancreatic & Acute pancreatitis, chronic pancreatitis \\
\hline \multirow{2}{*}{ Gastric } & Gastrectomy \\
\hline & Gastritis, pernicious anaemia, gastric ulcer \\
\hline \multirow{3}{*}{ Intestinal } & Intestinal parasites \\
\hline & Small Bowel Resection \\
\hline & $\begin{array}{c}\text { Inflammatory Bowel Diseases (IBD), Crohn's } \\
\text { Disease, Ulcerative Colitis }\end{array}$ \\
\hline \multirow{4}{*}{ Miscellaneous } & Malignancy \\
\hline & Connective tissue disease \\
\hline & Hyperthyroidism, Hypothyroidism \\
\hline & Menorrhagia, acute blood loss \\
\hline
\end{tabular}

Renal failure, thyroid disease, rheumatoid arthritis and malignancy (of any origin) were also identified as possible confounders that may cause anaemia, malnutrition and osteoporosis, and were excluded. Some of these conditions can be associated with coeliac disease and some of them are strongly associated, therefore, the emphasis was given on recognition of cases where an easily identifiable cause of symptoms was not always available. For example, conditions like 'osteoporoses' are categorised as 'consider testing' rather than 'offer testing'.

\section{Results}

Iron deficiency anemia (IDA) comprised of 68 admissions, of which 61 were diagnosed as IDA, while 17 were IBS. The patients in this subset came from all ages, the median age was 65.5 (10-90\% range $24-84$ years), including a mean age of 58.3 years $( \pm 22.4)$. About $50 \%$ of the IDA patients and $59 \%$ of the IBS patients had coeliac disease investigations, respectively.

In contrast, coeliac investigations were requested only for $20 \%$ of the patients from all other subsets. Patients admitted with unspecified anemia and protein-energy malnutrition (PEM) had a median age of 74.5 (10-90\% range 47-89 years) and 78 (10-90\% range $41-91$ years) years, respectively, and were relatively older than IDA (median $65.5,10-90 \%$ range 24-84 years) patients. A total of 106 unspecified anaemia admissions were observed, only $11 \%$ (12) were ordered for celiac investigations. On the other hand, $15.6 \%$ of the seventy-seven Protein Energy Malnutrition (PEM) admissions were requested for coeliac investigations (Table 2). While, coeliac testing is also recommended for patients diagnosed with Type 1 Diabetes, and Dermatitis Herpetiformis; no such diagnoses were made on the adult wards during the review period. 
Table 2. Type of testing for diagnosing coeliac disease of suspected patients admitted in the hospital

\begin{tabular}{|c|c|c|c|c|c|c|}
\hline Tests & $\begin{array}{c}{ }^{*} \mathrm{IDA}, \\
\mathrm{N},(\%)\end{array}$ & $\begin{array}{c}{ }^{*} \mathrm{UA}, \\
\mathrm{N},(\%)\end{array}$ & $\begin{array}{c}{ }^{*} \mathrm{PEM}, \\
\mathrm{N},(\%)\end{array}$ & $\begin{array}{c}{ }^{*} \mathrm{IBS}, \\
\mathrm{N},(\%)\end{array}$ & $\begin{array}{c}{ }^{*} \mathrm{OP}, \\
\mathrm{N}(\%)\end{array}$ & $\begin{array}{c}{ }^{*} \mathrm{~F} / \mathrm{B} 12 \mathrm{DA}, \\
\mathrm{N}(\%)\end{array}$ \\
\hline Histology only & $10(16.4)$ & $7(7.4)$ & $1(1.6)$ & $3(17.6)$ & $0(0.0)$ & $0(0.0)$ \\
\hline $\begin{array}{c}\text { Serology only } \\
\begin{array}{c}\text { Serology then } \\
\text { histology }\end{array}\end{array}$ & $2(3.3)$ & $1(1.05)$ & $4(6.3)$ & $1(5.9)$ & $0(0.0)$ & $0(0.0)$ \\
\hline Prior histology only & $8(13.1)$ & $3(3.1)$ & $1(1.6)$ & $5(29.4)$ & $1(5.0)$ & $0(0.0)$ \\
\hline Prior serology only & $2(3.3)$ & $3(3.1)$ & $3(4.8)$ & $0(0.0)$ & $2(10.0)$ & $0(0.0)$ \\
\hline $\begin{array}{c}\text { Prior histology } \\
\text { and serology }\end{array}$ & $2(3.3)$ & $2(2.15)$ & $3(4.8)$ & $0(0.0)$ & $0(0.0)$ & $0(0.0)$ \\
\hline $\begin{array}{c}\text { Prior histology, } \\
\text { current serology }\end{array}$ & $1(1.6)$ & $0(0.0)$ & $0(0.0)$ & $0(0.0)$ & $0(0.0)$ & $0(0.0)$ \\
\hline $\begin{array}{c}\text { Prior serology, } \\
\text { current histology }\end{array}$ & $1(1.6)$ & $1(1.05)$ & $0(0.0)$ & $0(0.0)$ & $0(0.0)$ & $0(0.0)$ \\
\hline $\begin{array}{c}\text { No investigations } \\
\text { Total number } \\
\text { of patients }\end{array}$ & $61(50.8)$ & $77(81.1)$ & $51(80.9)$ & $7(41.2)$ & $17(85.0)$ & $2(100)$ \\
\hline $\begin{array}{c}\text { Total number } \\
\text { of episodes }\end{array}$ & $65(100)$ & $63(100)$ & $17(100)$ & $20(100)$ & $2(100)$ \\
\hline \begin{tabular}{c} 
f \\
\hline
\end{tabular} & 106 & 77 & 17 & 26 & 2 \\
\hline
\end{tabular}

"IDA - Iron Deficiency Anaemia; UA - unspecified anaemia; PEM - protein energy malnutrition; IBS - irritable bowel syndrome; OP - osteoporosis; F / B12 DA - folate and B12 deficiency anaemia; "Prior" - prior to the audit period

Later, in the extended data review for the period between February 2012 and February 2013, we determined one year prevalence for coeliac testing. In this extended review we found that, 1088 coeliac serology tests were performed at Goulburn Valley Health and $5.2 \%$ of these serology tests were positive. Of the 57 positive serology results, thirty underwent a duodenal biopsy. Only fourteen new patients were diagnosed in this period and all of them were appropriately counseled and managed. One biopsy was performed to assess progress in a patient already known to have coeliac disease. A few of these tests were performed in the paediatric inpatient ward, where the majority was requested by general practitioners within the Hume Region. Duodenal biopsy specimens were submitted to the hospital histopathology laboratory for $52.6 \%$ of the 57 serology positive patients; $50 \%$ of these biopsies were diagnosed as positive coeliac disease cases. The remaining twenty-seven serology positive cases were managed externally. It is not known whether they were biopsied and reported at a different pathology services.

\section{Discussion}

The study reported that half of the IDA and $60 \%$ of the IBS patients, and only one fifth of all other suspected patients were requested for coeliac disease testing. The proportion of testing is very low especially for cases other than IBS or IDA. The NHS and WGO guidelines recommend coeliac testing in patients with localised gastrointestinal symptoms, or manifestations of Malabsorption. American College of Gastroenterology recommends a wide range of symptoms required coeliac testing [9] that may not be followed in many settings including rural Australia. Testing is mandatory in patients with objective biochemical evidence of Malabsorption, but clinical judgement is permitted when the clinical cues are more subjective.

The study highlighted low rates of testing in some categories of at-risk patients. Absence of a positive diagnosis in the six month period of review prompted a second extended review of twelve months for examining all serology requests, although this further enquiry does not completely relate to the question that the study seeks to address. Moreover, the exclusion of some of the medical conditions is likely to be a limitation of the study but hopefully does not detract from the main message that the study set out to convey.

The NHS guidelines recommend transglutaminase IgG serology in the IgA-deficient subpopulation, and equivalent IgA assays in all others [10]. The costly endomysial serology is reserved for intermediary titres. In cases where the ongoing clinical suspicion of coeliac disease is low, a normal result can obviate an invasive and expensive duodenal biopsy. All other patients need to attend a gastroenterology clinic, and undergo a duodenal biopsy to definitively diagnose or exclude coeliac disease. By contrast, endomysial and transglutaminase are equally acceptable assays, alone or in combination under WGO guidelines. Alternatively, clinicians may skip serology if the suspicion is high [5]. Therefore, serology, biopsy, or both investigations (in sequence) are acceptable under different circumstances.

The strong negative predictive values of serology and biopsy are predicated on adequate gluten intake in the preceding six weeks (more than one meal each day). Negative results are interpreted in their clinical context, and repeat serology and/or biopsy may be reasonable at a later date. Negative biopsies may be repeated in serologically positive patients after 1-2 years [5].

Acknowledging that these decisions rely on a gestalt of clinical clues that may be difficult to document or quantify, all investigated patients (Table 2) were, by WGO standards, appropriately managed. The emboldened cases violate stricter NHS guidelines, which forbid duodenal biopsy without prior serology. It is unclear how many duodenal biopsies could be avoided through prior serology and greater 
clinical acumen, as many serologically negative patients were also deemed to require biopsy. Duodenal biopsy is mandatory in serologically positive patients; however none were recruited in this population at initial review.

Australians with chronic mal-absorptive or gastrointestinal symptoms are initially assessed and investigated in the community setting [6]. A gastroenterologist would subsequently review selected patients to confirm the clinical indication, and perform the duodenal biopsy. In most cases, dietary gluten restriction definitively manages the disease. The tiered approach is motivated by the relative scarcity and expense of gastroenterologist consultations and duodenal biopsies, as compared to general practitioner visits and coeliac serology. Travel costs accentuate this difference in regional centres that rely on visiting specialists, or referrals to metropolitan centres. These additional penalties should incentivize stricter patient selection, possibly through highly specific and sensitive serological assays, which are widely available, and were underutilised in this review. Conversely, under-diagnosis in resource-poor settings exposes patients to additional symptomatic burden and malnutrition.

Professional development on this topic should emphasise the varied presentations of this important differential, and the role of serology and clinical judgement in its diagnostic algorithm. Such opportunities include case discussions, seminars, and periodic contact with gastroenterologists. General practitioners should have ready access to serology, endoscopy and histopathology services. Gastroenterologists should be available to advise and assist in difficult or refractory cases.

The hospital inpatient ward represents an acute caseload, in which coeliac disease is rarely the primary reason for admission. Habitual patterns could account for the relatively low testing rates found in this study. However, the regional hospitals should demonstrate leadership in developing and adhering to a guideline that optimally utilises the available resources. The adroit recognition of coeliac disease, with the relative proximity of endoscopy services, enables its concurrent and cost-effective management.

Regional areas would thus benefit from increased resources to recognise and diagnose coeliac disease. This might entail increased access to endoscopy and histopathology, following up of the results and specialist advice in difficult cases.

\section{Conclusions}

Training is crucial for practicing doctors to test appropriately and diagnose coeliac diseases. Continued medical education in this area may help to bring unknown cases under diagnosis and management. Although the findings may not be generalizable, however, it may be recommended that the rural and regional hospitals should have availability of specialist gastroenterology services as well as necessary investigations, so that referral by the general practitioners can be dealt with effectively for reducing morbidity. There can be a host of approaches including telemedicine support and dedicated pathology services.

\section{Acknowledgements}

Authors of this study acknowledge the contribution of Health Information Department of Goulburn Valley Base Hospital for their effort in data extraction.

\section{Conflicts of Interest}

The authors declare no conflicts of interest.

\section{REFERENCES}

[1] Rewers M, Epidemiology of celiac disease: what are the prevalence, incidence, and progression of celiac disease. Gastroenterology 2005. 128(4 Suppl. 1): p. S47-51.

[2] Catassi, C., S. Gatti, and E. Lionetti, World perspective and celiac disease epidemiology. Dig Dis, 2015. 33(2): p. 141-6.

[3] Hovell, C.J., et al., High prevalence of coeliac disease in a population-based study from Western Australia: a case for screening? Med J Aust, 2001. 175(5): p. 247-50.

[4] Van Heel, D.A. and J. West, Recent advances in coeliac disease. Gut, 2006. 55(7): p. 1037-46.

[5] J Bai, et al., WGO Practice Guidelines: Celiac Disease, W.G. Organisation, Editor. 2007.

[6] Gastroenterology Society Australia (GESA), Coeliac Disease, Digestive Health Foundation, Editor. 2007: Sydney.

[7] Julio C. Bai, M.F., Gino Roberto Corazza, Detlef Schuppan, Michael Farthing, Carlo Catassi, Luigi Greco, Henry Cohen, Carolina Ciacci, Alessio Fasano, Andrea González, Justus H. Krabshuis, Anton LeMair, World Gastroenterology Organisation Global Guidelines: Celiac disease, World Gastroenterology Organisation, Editor. 2012, World Gastroenterology Organisation, 2012.

[8] National Institute for Health and Care Excellence, Coeliac disease: Recognition and assessment of coeliac disease, Manchester National Institute for Health and Clinical Excellence, Editor. 2009.

[9] Rubio-Tapia, A., et al., ACG clinical guidelines: diagnosis and management of celiac disease. Am J Gastroenterol, 2013. 108(5): p. 656-76; quiz 677.

[10] Ludvigsson, J.F., et al., Diagnosis and management of adult coeliac disease: guidelines from the British Society of Gastroenterology. Gut, 2014. 63(8): p. 1210-28. 